\title{
Una comparación cualitativa de la dinámica de sistemas, la simulación de eventos discretos y la simulación basada en agentes
}

\author{
Alfonso Sarmiento Vásquez* \\ Universidad de La Sabana. Bogotá, Colombia
}

Eduardo López Sandoval*

Universidad de Lima. Perú

Recibido: 11 de mayo del 2017 / Aprobado: 20 de junio del 2017

RESUMEN: La dinámica de sistemas, la simulación de eventos discretos y la simulación basada en agentes son tres formas de modelamiento ampliamente usadas para la toma de decisiones empresariales. Estos paradigmas están enfocados en representar el comportamiento de los sistemas en el tiempo, aunque con perspectivas distintas. En este artículo se presenta una comparación de los tres paradigmas en 10 aspectos, comparación que puede servir como referente para elegir la técnica más apropiada para un problema requerido.

Palabras clave: teoría de sistemas / métodos de simulación / modelos matemáticos

\section{A qualitative comparison of system dynamics, discrete event simulation, and agent-based simulation}

AвsTRACT: Systems dynamics, discrete event simulation, and agent-based simulation are three widely used forms of modeling for business decision making. These paradigms are focused on representing the behavior of systems over time, although in different perspectives. This paper compares the three paradigms in 10 aspects and it can serve as an indicator to choose the most appropriate technique to a required problem.

Keywords: system theory / simulation methods / mathematical models

* Correos electrónicos: alfonsosava@unisabana.edu.co, elopez@ulima.edu.pe 


\section{INTRODUCCIÓN}

La simulación se puede definir como la imitación de un sistema usando un modelo de computadora con el fin de evaluar y mejorar el desempeño de este (Harrell, Ghosh y Bowden, 2004). Un sistema se entiende como una colección de elementos que funcionan juntos para conseguir una meta deseada (Blanchard, 1991). El estado de un sistema en un punto específico de tiempo se indica por medio de las variables de estado.

Hay muchas formas de organizar los modelos de simulación. Una de las más comunes es dividirlos en los siguientes tipos: (a) estáticos y dinámicos, (b) determinísticos y estocásticos, y (c) continuos y discretos (Law, 2014; Harrell et al., 2004).

Un modelo de simulación estático es una representación de un sistema en un punto particular de tiempo o uno que se use para representar un sistema que no está basado en el tiempo (Law, 2014). Por ejemplo, simular la salida de un decodificador por la entrada de diversos códigos generados de forma aleatoria. Por otro lado, un modelo dinámico de simulación incluye el paso del tiempo. Un mecanismo de reloj se mueve hacia adelante en el tiempo y las variables de estado son actualizadas conforme el tiempo avanza. Este tipo de simulación es adecuado para analizar sistemas de manufactura y servicios, ya que estos operan a través del tiempo.

Un modelo de simulación que no contiene componentes probabilísticos o aleatorios se denomina determinístico; por ejemplo, un sistema de ecuaciones que describa una reacción química. En este tipo de modelos, una vez que se han definido los datos de entrada y el estado inicial, todos los estados futuros son determinados con certeza. Algunos sistemas, sin embargo, pueden ser modelados con algunos componentes aleatorios, como en el caso de los modelos de simulación estocásticos. Estos tienen entradas aleatorias que generan también resultados aleatorios. Los sistemas de colas y muchos modelos de inventarios se configuran de esta forma.

En un modelo de simulación continuo el estado del sistema es representado por variables dependientes que cambian continuamente con el paso del tiempo. Este tipo de modelo se construye definiendo las ecuaciones para un conjunto de variables de estado cuyo comportamiento dinámico simula el sistema real, por ejemplo, el flujo de agua a través de una tubería. En los modelos de simulación discretos, las variables de estado cambian instantáneamente en puntos separados de tiempo. En 
este caso, se dice que el sistema solamente puede cambiar en un número contable de puntos en el tiempo; por ejemplo, una línea de producción con una máquina, en la que el tiempo se contabiliza al iniciar un trabajo en la máquina y al finalizarlo. No se toma en consideración el paso del tiempo entre estos dos instantes, ya que la variable de estado que controla la situación de la máquina no cambia durante el procesamiento del trabajo.

La literatura menciona tres importantes paradigmas ${ }^{1}$ de simulación: (1) dinámica de sistemas, (2) simulación de eventos discretos y (3) simulación basada en agentes (Borshchev, 2013; Behdani, 2012). Cada uno de estos tres paradigmas tiene su grupo de seguidores, publicaciones en revistas y conferencias independientes que soportan sus actividades.

Algunos autores citan un cuarto paradigma, denominado sistemas dinámicos (Serova, 2013; Yu, 2008). Este paradigma es utilizado especialmente en disciplinas de ingeniería técnica, como mecánica, eléctrica y química como una parte estándar de su proceso de diseño. Dicho paradigma sirve para diseñar y modelar el comportamiento de sistemas físicos a lo largo del tiempo por medio de un número de variables de estado y de sistemas de ecuaciones diferenciales de varias formas sobre esas variables (Borshchev y Filippov, 2004). Las variables de estado tienen significados físicos directos: velocidad, aceleración, presión, concentración, etc. Por ejemplo, se usan para estudiar la dinámica de los aviones o los sistemas de control en los automóviles. En este artículo se presta especial atención a las tres principales técnicas de la simulación cuyo enfoque predominante son los procesos de negocio y los sistemas sociales. No se incluyen en este análisis los sistemas dinámicos.

La visión en que se centra cada uno de los tres paradigmas de simulación está asociada a las características de los modelos de simulación mencionados líneas arriba. Si bien los tres paradigmas trabajan con modelos dinámicos de simulación, el paradigma de dinámica de sistemas trabaja con modelos continuos y determinísticos, mientras que los otros dos paradigmas lo hacen con modelos discretos y estocásticos.

\section{ANÁLISIS CUALITATIVO DE LOS MÉTODOS DE SIMULACIÓN}

En este trabajo de investigación se hace una descripción detallada de cada uno de los tres paradigmas de simulación, se explica tanto el marco

1 Un paradigma está caracterizado por un conjunto de supuestos básicos y algunos conceptos subyacentes en los que se basa para describir el mundo. 
conceptual que los define como el conjunto de supuestos y aspectos clave que los caracterizan en su visión del mundo. Finalmente, se organizan los elementos distintivos de los tres paradigmas en un cuadro comparativo para poder contrastarlos con los requisitos de modelamiento de futuros sistemas de estudio.

\subsection{Dinámica de sistemas}

Jay Forrester (1958) introdujo la dinámica de sistemas o system dynamics (SD) a finales de la década de 1950 como una metodología de modelamiento y simulación para el análisis y toma de decisiones a largo plazo sobre problemas empresariales industriales. SD tiene su origen en la administración y la ingeniería de control. El paradigma usa una perspectiva basada en la realimentación y en los retrasos de la información para entender el comportamiento dinámico de complejos sistemas físicos, biológicos y sociales (Angerhofer y Angelides, 2000). La idea esencial en SD es que todos los elementos en un sistema interactúan a través de relaciones causales. Estas relaciones son representadas por bucles de realimentación, los cuales controlan las interacciones entre los elementos del sistema y son la causa de su comportamiento (Rabelo et al., 2003).

El modelamiento en SD se puede dividir en dos fases, cualitativa y cuantitativa. Estas se detallan a continuación.

\subsubsection{Fase de modelamiento cualitativo}

En esta etapa se definen las relaciones de causalidad que se producen entre los elementos que integran el sistema. Esto se logra elaborando el diagrama causal, que es una herramienta visual para representar las estructuras de realimentación de los sistemas (Sterman, 2000). Un diagrama causal consiste en variables conectadas por flechas que denotan las influencias causales entre estas variables.

Existen dos tipos de causalidad: positiva (+) y negativa (-). El carácter de la relación se expresa asociando un signo a la flecha. En una relación positiva entre dos variables $\mathrm{X}$ e $\mathrm{Y}$, si X se incrementa (o por el contrario disminuye), entonces $\mathrm{Y}$ se incrementa (o disminuye) en una cantidad por encima (o por debajo) de la que hubiera mostrado si no hubiera ocurrido el cambio en X. La figura 1 muestra una relación de causalidad positiva, en la que un mayor incremento en el presupuesto de mercadeo genera un aumento en las ventas. 


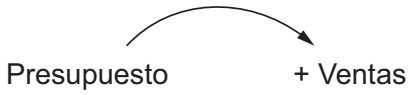

de mercadeo

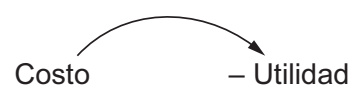

Figura 1. Tipos de causalidad

Elaboración propia

Por otro lado, en una relación negativa entre dos variables X e Y, si X se incrementa (o por el contrario disminuye), entonces $\mathrm{Y}$ disminuye (o se incrementa) en una cantidad por debajo (o por encima) de la que hubiera mostrado si no hubiera ocurrido el cambio en X. La figura 1 muestra una relación de causalidad negativa, en la que se interpreta que a un mayor costo, la utilidad se reduce.

Cuando un grupo de variables interconectadas por relaciones causales forman un camino cerrado que comienza en una variable inicial y acaba en esa misma variable, entonces se dice que este grupo de variables forman un bucle de realimentación. Existen dos tipos de bucles: de realimentación positiva o de refuerzo $(\mathrm{R})$ y de realimentación negativa o de balance (B).

Un bucle $\mathrm{R}$ genera un comportamiento de crecimiento o decrecimiento del sistema, que lo aleja del punto de equilibrio; es decir, tiende a desestabilizar los sistemas de forma exponencial. Por ejemplo, en la figura 2 las variables Nacimientos y Población forman un bucle. Así, cuando hay un mayor número de nacimientos (considerando una tasa de natalidad constante), se produce un aumento en la población, lo que genera a su vez que se incremente el número de nacimientos. Lo mencionado anteriormente origina un crecimiento exponencial de la variable Población.

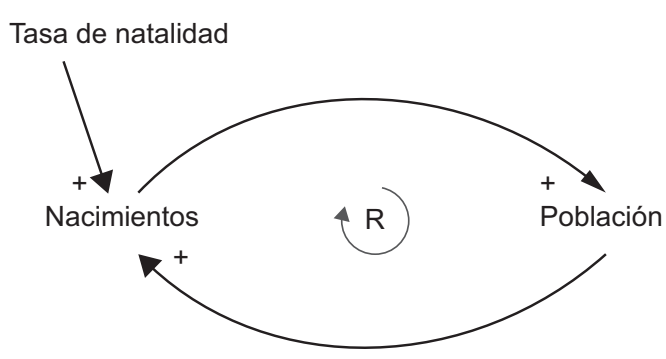

Figura 2. Bucle de refuerzo Elaboración propia

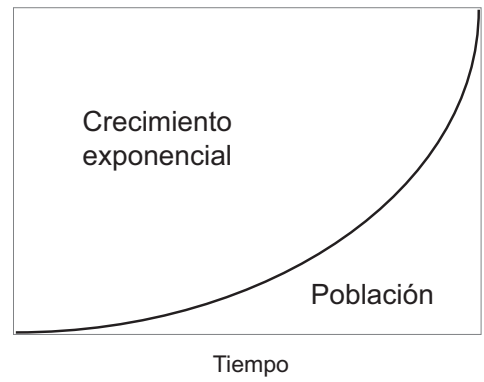

Tiempo 
Un bucle B se conoce como estabilizador o regulador, y en él una variación de un elemento se transmite a lo largo del bucle de manera que genera un efecto que contrarresta la variación inicial. Por ejemplo, en la figura 3 las variables Población y Muertes forman un bucle que se interpreta así: ante el incremento de la población, se produce un aumento en el número de muertes, lo que genera a su vez (considerando una tasa de mortalidad constante) que disminuya la población. Esto origina que la variable Población tienda a buscar asintóticamente un equilibrio.
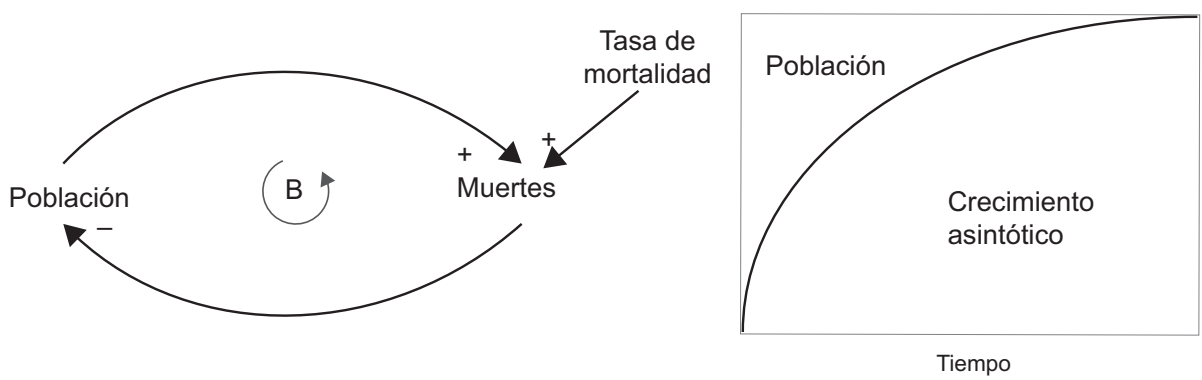

Figura 3. Bucle de balance Elaboración propia

Una regla que se suele utilizar para determinar la polaridad de un bucle de realimentación consiste en multiplicar las polaridades de las relaciones individuales que lo constituyen (Richardson, 1995). Los bucles con polaridad positiva son de refuerzo y los de polaridad negativa son de balance. En el ejemplo de la figura 3, la relación Población $\rightarrow$ Muertes tiene una polaridad positiva y la relación Muertes $\rightarrow$ Población tiene una polaridad negativa. El producto de ambas relaciones da una polaridad negativa, lo que indica balance.

\subsubsection{Fase de modelamiento cuantitativo}

Para poder generar un modelo cuantitativo que se pueda simular en un computador, se debe traducir el diagrama causal a un diagrama de Forrester. Este es un paso intermedio para la obtención de las ecuaciones matemáticas que definen el comportamiento del sistema. El diagrama de Forrester tiene dos elementos: parámetros (o constantes) y variables. Las variables pueden ser de tres tipos (Morlán, 2010):

- Variables de nivel. Definen el estado del sistema y acumulan magnitudes con el tiempo. 
- Variables de flujo. Representan el cambio de las variables de nivel durante un periodo de tiempo. Son las derivadas de los niveles con respecto al tiempo.

- Variables auxiliares. Se utilizan para descomponer ecuaciones complejas en ecuaciones más simples con el fin de que se facilite la lectura el modelo.

En la figura 4 se muestra un ejemplo del diagrama de Forrester, donde Población es una variable de nivel, Nacimientos y Muertes son variables de flujo, y tasas de natalidad y de mortalidad son parámetros. Las dos nubes (a la izquierda de la variable Nacimientos y a la derecha de la variable Muertes) representan una fuente y un sumidero, respectivamente, es decir, marcan los límites del modelo. En este caso, no interesa analizar de dónde vienen los nacimientos ni adónde van las muertes.

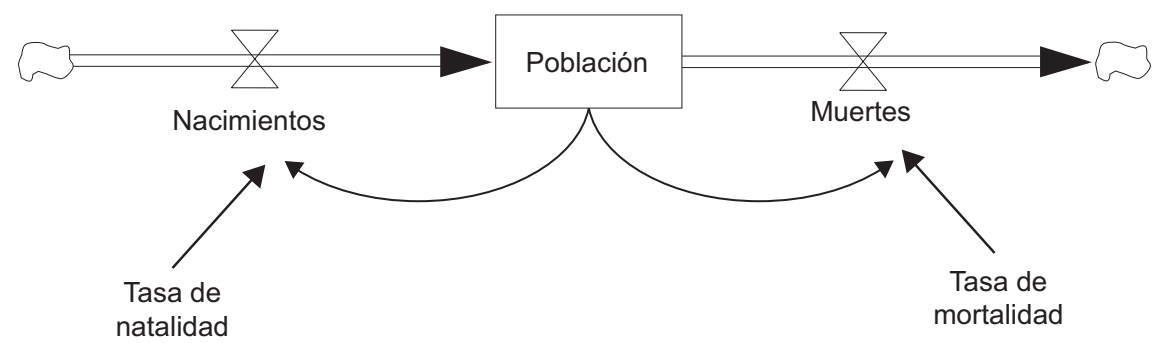

Figura 4. Variables del diagrama de Forrester Elaboración propia

El valor de las variables de nivel y de flujo se obtiene de las siguientes ecuaciones:

- Ecuaciones de nivel. En estas ecuaciones se calcula el valor de las variables de nivel como una acumulación en el tiempo de la diferencia de los flujos de entrada y salida de un proceso. Por ejemplo, el valor de Población en el instante de tiempo $t$ se puede expresar como la integral de la diferencia entre Nacimientos y Muertes, partiendo de un valor inicial de Población en el instante $t=0$.

$$
\text { Población }(\mathrm{t}) \text { = Población }(0)+\int_{0}^{\mathrm{t}}[\mathrm{Nacimientos}(\mathrm{z})-\operatorname{Muertes}(\mathrm{z})] \mathrm{dz}
$$


- Ecuaciones de flujo. A diferencia de las variables de nivel, las ecuaciones de flujo no cuentan con una forma estándar. Por ejemplo, los nacimientos se pueden expresar como un porcentaje de la población dado por la tasa de natalidad.

$$
\text { Nacimientos }(t)=\text { Población }(t) \times \text { tasa de natalidad }
$$

Los diagramas de Forrester representan sistemas continuos; sin embargo, su simulación es discreta, ya que se realiza por medio de un computador. Esto significa que en lugar de que se manejen diferenciales de tiempo $d t$, se utilizan incrementos o intervalos discretos de tiempo $\Delta t$.

A continuación, se listan las principales características de SD (Dangerfield, 2014; Brailsford, Desai y Viana, 2010):

- El principio fundamental en SD indica que la estructura del sistema determina su comportamiento a lo largo del tiempo. En otras palabras, la manera en que los componentes separados de cualquier sistema se relacionan y afectan entre ellos determina el comportamiento emergente del sistema como una totalidad. El componente básico usado en la construcción de un modelo es el bucle de realimentación.

- El paradigma trabaja con valores agregados (de productos, gente, etc.) y no con entidades individuales. Los objetos almacenados (como un todo) en el mismo nivel se consideran homogéneos e indistinguibles. Se dice que con la técnica de SD se tiene una visión del bosque sin poder identificar cada uno de los árboles, es decir, se representan los objetos o sus características mediante propiedades promedio. Esto es un resultado directo de la técnica de modelamiento usada, en la que las variables de nivel son acumulaciones de los flujos, lo que no permite identificar los objetos simples fluyendo a través del sistema.

- Es esencialmente un método determinístico y no maneja la variabilidad de manera muy efectiva, a pesar de los esfuerzos de los desarrolladores de software por incluir en este funciones de probabilidad.

- Los modelos de SD corren de forma muy rápida y, al ser determinísticos, no requieren de múltiples replicaciones.

- Representaciones lineales ${ }^{2}$ son usadas en los modelos de SD cuando se convierte una unidad de medida en otra unidad de medida (por ejemplo, años en meses) o cuando la relación entre las variables es

2 Cuando una variable se incrementa en una proporción constante con respecto a otra variable, tal como $\mathrm{y}=\mathrm{mx}+\mathrm{b}$. 
esencialmente constante en el intervalo en que se está estudiando. Sin embargo, SD da al modelador la flexibilidad de introducir relaciones no lineales en el modelo.

- Hace posible el análisis de cómo las relaciones no lineales de causalidad entre las variables y los parámetros del modelo influyen en el comportamiento del sistema. Este análisis, generalmente, no se puede obtener mediante las técnicas de correlación, ya que estas sirven para detectar únicamente relaciones lineales.

- Es una metodología de arriba abajo (top-down), es decir, tiene un enfoque macroscópico del mundo, similar a una síntesis holística de un sistema de elementos que están dinámicamente interconectados. Se considera que tiene un planteamiento más estratégico, en el cual los eventos y las decisiones son percibidos como formas de comportamiento y estructuras del sistema.

- Se enfoca en la dinámica del sistema por causas endógenas. En concreto, los cambios en el tiempo se dan en los límites internos del sistema, debido a las interacciones de los componentes, aunque el estímulo inicial de estas dinámicas puede haber sido exógeno (externo a los límites del sistema).

- Pertenece a la clasificación de simulación continua, en la cual se asume que los flujos cambian de forma continua con respecto al tiempo y son regidos por ecuaciones diferenciales de primer orden ${ }^{4}$.

- Aunque las tasas de los flujos son incluidas, los modelos de SD se concentran principalmente en el comportamiento de los niveles o acumuladores en el sistema. Estos son descritos por ecuaciones de integración.

- No se ignoran las variables blandas (como moral o reputación), que tienen una influencia causal en el sistema.

\subsection{Simulación de eventos discretos}

La simulación de eventos discretos, en inglés discrete event simulation (DES), se llevó a cabo en la década de 1960 en las áreas de investigación

3 Método que empieza en el nivel conceptual más alto y desciende hasta los detalles.

4 Ecuaciones en las que intervienen derivadas de primer orden, es decir, de la forma $\mathrm{dy} / \mathrm{dx}$. 
de operaciones e ingeniería industrial para ayudar a analizar y mejorar los procesos industriales y de negocios. Cabe destacar el desarrollo del software GPSS (General Purpose Simulation System) por Geoffrey Gordon de IBM, que ha contribuido conceptualmente al desarrollo de los lenguajes comerciales de DES que surgieron desde entonces.

DES se refiere a la modelación computacional de sistemas que evolucionan en el tiempo mediante cambios instantáneos en las variables de estado. Los cambios ocurren en puntos separados del tiempo (o intervalos discretos) accionados por eventos. Es decir, el modelo salta desde el tiempo de un evento hasta el tiempo del siguiente evento. Estas características otorgan a DES flexibilidad y eficiencia para que pueda ser usada en una gran variedad de problemas.

En términos simples, DES modela el progreso de los sistemas de colas a lo largo del tiempo. Esto lo consigue representando el mundo como un conjunto de entidades que fluyen a través de una red de colas y actividades, donde los recursos son compartidos por las actividades (Robinson, 2014). En otras palabras, DES es muy útil para problemas que consisten en simulaciones de colas o redes complejas con colas, en las cuales los procesos pueden ser bien definidos y el énfasis está en representar incertidumbre a través de distribuciones estocásticas. Muchas de estas aplicaciones se dan en las industrias de manufactura y de servicios (Siebers, Macal, Garnet, Buxtony y Pidd, 2010).

Los conceptos principales de DES son entidades, atributos, variables, eventos, recursos, colas y tiempo (Karnon et al., 2012; Kelton, Sadowski y Sturrock, 2006).

- Entidades. Son objetos dinámicos que tienen atributos, consumen recursos y entran a las colas. Las entidades cambian de estado, afectan y son afectadas por otras entidades, así como por el estado del sistema. Usualmente, son creadas, se mueven por el sistema durante un lapso de tiempo y luego son eliminadas al salir de él, aunque puede haber entidades que nunca dejen el sistema y se mantengan circulando de forma permanente.

- Atributo. Es una característica común de todas las entidades, pero con un valor específico que puede variar de una entidad a otra (por ejemplo, edad, sexo, color, etc.). Estos valores pueden ser usados para determinar cómo una entidad responde a ciertas circunstancias, además, pueden ser modificados durante cualquier momento de la simulación. 
- Variables. Representan características del sistema que son independientes de los tipos de entidades, sin embargo, estas últimas pueden modificar las variables.

- Evento. Es algo que sucede en un instante de tiempo y que puede cambiar los atributos, las variables o las estadísticas.

- Recurso. Es un objeto que provee un servicio a una entidad. Los recursos representan personas, equipo, espacio físico, etc. La entidad captura unidades de un recurso cuando está disponible y las libera cuando termina de usarlo.

- Colas. Si un recurso está ocupado cuando una entidad lo requiere, entonces la entidad debe esperar y formar una cola. Las colas pueden tener capacidades máximas y reglas que definen la prioridad de las entidades en la cola, como FIFO (first in first out).

- Tiempo. Un reloj de simulación mantiene un registro del tiempo. A diferencia del tiempo real, el reloj de simulación no calcula todos los valores de forma continua, sino que salta del tiempo de un evento al tiempo del siguiente evento. Para ello, el reloj de simulación interactúa con el calendario de eventos (que es una lista de los eventos programados).

Los modelos de simulación discreta pueden desarrollarse a partir de tres enfoques (Heath, Brailsford, Buss y Macal, 2011; Pegden, 2010; Pidd, 2004):

- Enfoque de eventos. El sistema es modelado al definir los cambios que ocurren al momento de los eventos. La tarea del modelador consiste en determinar los eventos que pueden cambiar el estado del sistema (el estado del sistema no cambia entre eventos) y luego desarrollar la lógica asociada a cada tipo de evento. Una simulación del sistema se produce al ejecutar la lógica que se relaciona con cada evento en una secuencia ordenada en el tiempo.

Deben plantearse las siguientes preguntas para iniciar el modelamiento de un sistema a partir de este enfoque:

- ¿Cómo se define el estado del sistema?

- ¿Cuáles son los eventos que pueden cambiar el estado del sistema?

- ¿Cuál es la lógica dentro de cada evento que define los cambios de estado?

Un modelo de gráfico por eventos se muestra en la figura 5 con un evento de arribo (con tiempo entre arribos $t_{a}$ ), una operación de 
servicio (con tiempo de servicio $t_{s}$ ) representada por un evento de inicio y un evento de fin, una cola $\mathrm{Q}$ inicialmente vacía, y un recurso $\mathrm{S}$ con una capacidad de $k$ unidades.

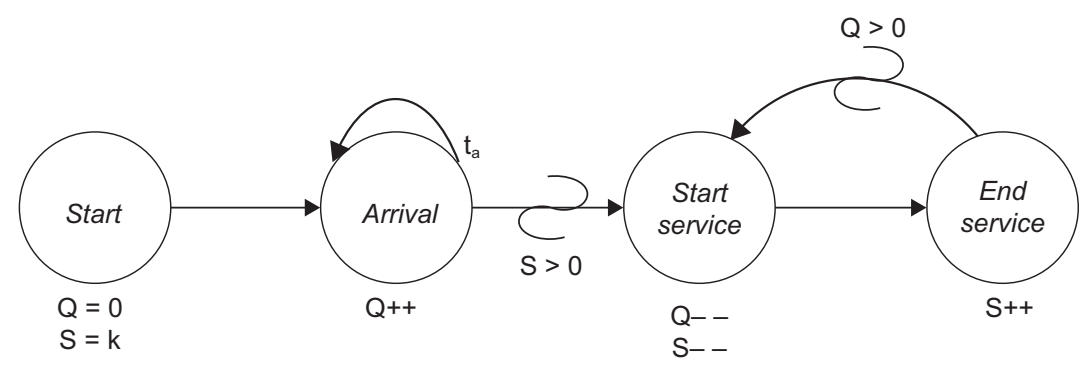

Figura 5. Modelo de gráfico por eventos para un sistema de colas Fuente: Kesaraju y Ciarallo (2012)

- Enfoque de actividades. Se originó como un método denominado escaneo de actividades. En este enfoque el modelador describe las actividades en que participan las entidades del sistema y determina las condiciones que causan que una actividad se inicie o finalice. Los eventos que comienzan o terminan la actividad no son programados por el modelador, pero son ejecutados por las condiciones especificadas para la actividad. Mientras el tiempo de la simulación avanza, se monitorean las condiciones para iniciar o finalizar una actividad. Si las condiciones son cumplidas, las acciones apropiadas para la actividad son ejecutadas. Para asegurar que cada actividad sea considera$\mathrm{da}$, es necesario monitorear el conjunto de actividades cada vez que el tiempo avance; por tal razón, este enfoque es menos eficiente que el de eventos discretos.

El método de escaneo de actividades evolucionó hacia el denominado método de las tres fases, que es una versión que mejora la eficiencia de la ejecución. Este procedimiento categoriza las actividades en B y C. Las actividades B están ligadas a la ocurrencia de un suceso y pueden ser programadas con anticipación, mientras que las actividades $\mathrm{C}$ se producirán cuando se verifiquen ciertas situaciones o condiciones.

En la figura 6 se muestra un modelo de diagrama cíclico de actividades, en inglés activity cycle diagram (ACD), para un sistema de colas. 


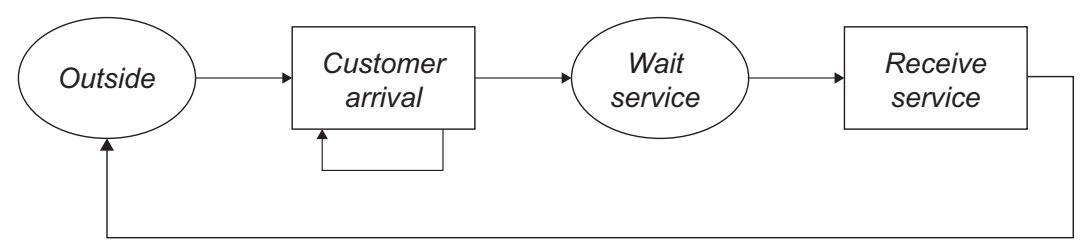

Figura 6. Modelo de diagrama cíclico de actividades para un sistema de colas Fuente: Robinson (2004)

- Enfoque de procesos. Muchos modelos de simulación incluyen secuencias de elementos que ocurren en patrones definidos a lo largo del tiempo; por ejemplo, una cola donde las entidades esperan para ser procesadas por el servidor. La lógica asociada con tal secuencia de eventos puede ser generalizada y definida por una única declaración, luego un lenguaje de simulación puede traducir tales declaraciones en una secuencia de eventos apropiada, y un lenguaje enfocado a los procesos emplea estas declaraciones para modelar el flujo de entidades a través del sistema. Asimismo, ya que una actividad se puede definir como el estado de un objeto entre dos eventos, entonces el enfoque por procesos se puede concebir como una sucesión continua de una o más actividades (Vangheluwe, 2001). La mayoría de los programas comerciales de DES trabajan con este enfoque (Heath et. al, 2011).

Se deben plantear las siguientes preguntas para iniciar el modelamiento de un sistema por este enfoque:

- ¿Cuáles son las entidades que se mueven a través del sistema?

- ¿Qué procesos son ejecutados cuando la entidad se mueve en el sistema?

Un modelo del enfoque por procesos se muestra en la figura 7. Es un flujograma en el que las entidades entran al proceso en el primer paso y lo abandonan en el último paso.

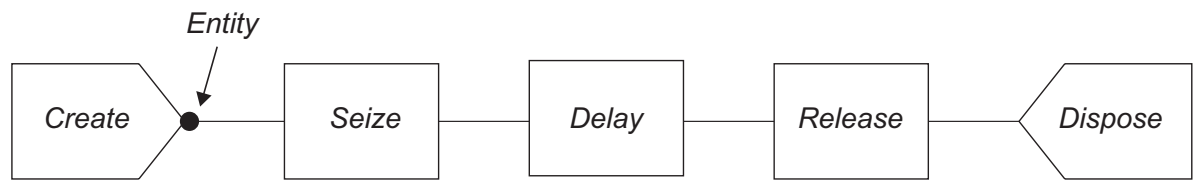

Figura 7. Modelo del enfoque por procesos para un sistema de colas Fuente: Kesaraju y Ciarallo (2012) 
En la figura 8 se representan los tres enfoques para un sistema que consiste en un cajero que atiende a los clientes, quienes llegan uno a la vez. Si el cajero no está disponible, los clientes se forman en una cola, respetando el orden de llegada. Una vez terminado el servicio, los clientes abandonan el sistema.

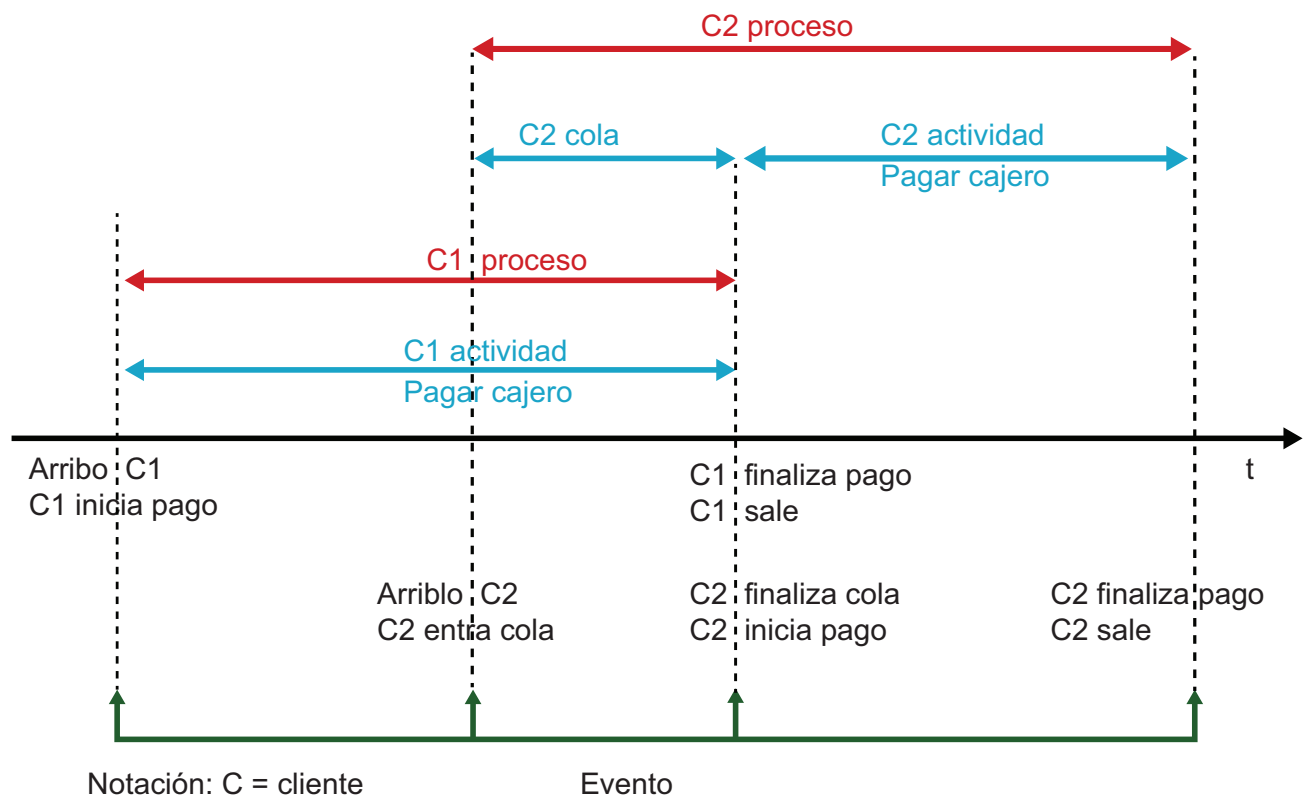

Figura 8. Sistema de colas con los tres enfoques Fuente: adaptado de Vangheluwe (2001)

Los modelos de DES se caracterizan por tener componentes estocásticos que imitan la naturaleza probabilística del sistema en estudio. Esa aleatoriedad es representada frecuentemente con variables aleatorias. La obtención de las funciones de probabilidad de dichas variables se conoce como el análisis de los datos de entrada.

Debido al carácter aleatorio de las variables de entrada del modelo, las medidas de desempeño que se toman al final de la simulación también tienen carácter aleatorio. Por ello, con el fin de que los resultados de la simulación tengan algún significado, deben emplearse técnicas estadísticas apropiadas para diseñar y analizar los experimentos (Law, 2014); por ejemplo, se recomienda hacer inferencias basadas en los intervalos de confianza de las medidas de desempeño más que en estimaciones puntuales de dichas variables. 
A continuación, se listan las principales características de DES (Brailsford, 2014b; Marshall, Burgos-Liz e Ijzerman, 2015a; Robinson, 2014; Skoogh y Johansson, 2008):

- Un elemento clave en casi todos los modelos de DES es la necesidad de representar la aleatoriedad del sistema en estudio; por tanto, se puede decir que DES es estocástica por naturaleza.

- Pertenece a la clasificación de simulación discreta, en la que el estado del sistema solamente puede cambiar en puntos discretos de tiempo, en los que ocurren eventos.

- Es una metodología de arriba abajo (top-down), es decir, el modelador especifica todas la reglas del sistema con anticipación y se mira de arriba abajo cómo se comportan los individuos (o entidades) a lo largo del tiempo. Estos supuestos pueden estar basados en datos históricos o en opiniones de expertos, y en DES están programados en las actividades. Por tanto, las condiciones en que las entidades siguen una ruta en particular o pasan una cierta cantidad de tiempo realizando una actividad tienen que ser codificadas en el modelo.

- Los sistemas son típicamente de bucle abierto (open-loop), por lo que la retroalimentación no juega un rol preponderante.

- Se hace uso frecuente de animaciones y gráficos, los cuales son muy útiles para comunicarse con los clientes.

- En el análisis de los datos de entrada y salida de los modelos de simulación se requiere conocimientos de estadística. En un proyecto de DES, la administración de los datos de entrada es la fase más importante y de mayor consumo de tiempo. Más del $30 \%$ del tiempo del proyecto se usa en la recolección de los datos, su transformación en información y el suministro de las entradas en el modelo de simulación. Asimismo, representaciones creíbles de parámetros estocásticos en simulaciones dinámicas requieren un número extenso de datos. Por otro lado, también es necesario el uso de métodos estadísticos para el análisis de los resultados. Se deben tomar decisiones acerca del número de replicaciones independientes que se requieren, la duración de la corrida de simulación, el uso de técnicas de reducción de varianza, la selección de los escenarios, etc.

- Las entidades se consideran objetos pasivos (sin inteligencia o capacidad para tomar decisiones), además, poseen atributos o características que determinan su comportamiento dentro del sistema y la manera en que interactúan con las otras entidades y el entorno. 
Sin embargo, el término entidad pasiva alude a que la secuencia de las entidades está dominada por reglas globales preestablecidas; es decir, aunque las entidades pueden elegir recorrer ciertos flujos del proceso (por ejemplo, de acuerdo con el valor de atributos determinados) esto no elimina el hecho de que siguen las reglas de un sistema centralizado. En consecuencia, no se tiene la facilidad para representar decisiones descentralizadas en DES. Así, una vez que una entidad ha empezado a procesarse en una actividad, es muy difícil interrumpir el procesamiento basándose en cambios del entorno. Justamente, esto dificulta modelar la complejidad del comportamiento en ambientes sociales, como en el caso en que las entidades representan a clientes y manifiestan su opinión sobre un fabricante o una marca específica, lo cual influye en la decisión de otros clientes sobre dicho fabricante o marca.

\subsection{Simulación basada en agentes}

La simulación basada en agentes, en inglés Agent-Based Simulation (ABS), se introdujo a principios de la década de 1990. Aunque en sus inicios este método fue meramente un tópico académico, durante el periodo 2000-2003 empezó a ser usado en simulación (Sumari, Ibrahim, Zacaria y Ab Hamid, 2013). Entre las razones de su impulso se encuentran las siguientes:

- El deseo de obtener una visión más profunda de los sistemas cuyo comportamiento no ha sido capturado de forma adecuada por los métodos de modelamiento tradicionales.

- Los avances en la tecnología de modelado procedentes de la informática, como el modelado orientado a objetos, el lenguaje UML (Unified Modeling Language) y los gráficos de estado.

- El rápido crecimiento en la disponibilidad de computadoras con CPU y memoria más potentes ya que los modelos de simulación basados en agentes son más exigentes en cálculos computacionales que los usados en la dinámica de sistemas y en la simulación de eventos discretos.

Los agentes son objetos que tienen estas características (Behdani, 2012):

- Autonomía. Tienen un cierto nivel de autonomía que les permite tomar decisiones sin un controlador central. Para lograr esto, cuentan 
con un conjunto de reglas que determinan su comportamiento. Otra forma de tomar decisiones es probabilistamente, al usar distribuciones de probabilidad para regir el proceso de decisión.

- Reactividad. Son capaces de percibir los cambios en el entorno (o ambiente) y en otros agentes; luego, responder a esos cambios con sus propias acciones cuando sea necesario.

- Proactividad. Tienen capacidad proactiva, es decir, no solo actúan en respuesta a los cambios que se han producido en su entorno, sino que también tienen sus propios objetivos.

- Habilidad social. Poseen la capacidad social para interactuar y comunicarse entre sí.

- Adaptabilidad. Pueden tener memoria para aprender y adaptar sus comportamientos, basados en la experiencia.

La forma en que interactúan los agentes se puede apreciar en la figura 9 .

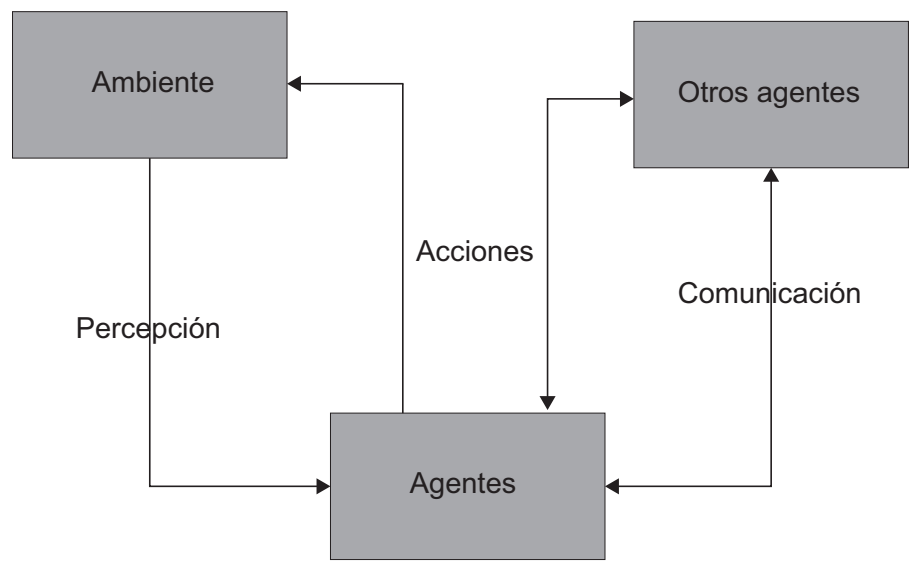

Figura 9. Interacción entre agentes

Fuente: adaptado de Salamon, T. (2011)

Este paradigma sugiere al modelador que vea el sistema de otra forma. ABS es muy útil cuando no se conoce cómo funciona el sistema en su conjunto o no se pueden identificar las variables claves y su interrelación, o no se distinguen claramente los flujos de proceso; sin embargo, sí se tiene una idea de cómo los objetos del sistema se comportan de forma individual. Algunas veces se puede dejar que los agentes 
interactúen entre ellos; otras veces se puede dejar a los agentes en un ambiente que tiene su propia dinámica. En cualquiera de los casos, el comportamiento global del sistema emerge de entre muchos (decenas, cientos, miles, incluso millones) comportamientos individuales concurrentes (Borshchev y Filippov, 2004).

A continuación, se describen las principales características de ABS (Brailsford, 2014; Schieritz y Milling, 2003; Macal y North, 2006):

- El principio fundamental en ABS indica que la interacción entre los agentes determina el comportamiento del sistema. Este paradigma se enfoca en las reglas de los agentes, las cuales determinan cómo se darán las interacciones y, por tanto, el comportamiento del sistema como un todo. Por ello, el componente básico usado en la construcción de un modelo es el agente, el cual es considerado entidad "activa", ya que puede tomar decisiones autónomas no preestablecidas.

- Es un método de abajo hacia arriba (bottom-up) ${ }^{5}$, es decir, es la fuente de un fenómeno denominado que emerge. Este ocurre cuando las interacciones entre objetos en un nivel dan como resultado objetos en otro nivel. En ABS este fenómeno no es modelado previamente como en SD o DES, en las que se imponen reglas globales al sistema. En ABS no existen reglas que rijan el sistema, sino que por el contrario el comportamiento de este emerge a lo largo de la simulación. Lo que sí se debe hacer es describir los comportamientos individuales de los agentes, que están basados en supuestos de cómo los individuos responden a estímulos de otros agentes y del entorno.

- ABS es una técnica adecuada cuando el problema requiere un fuerte modelamiento en aspectos espaciales o geográficos (posición, tamaño, velocidad, etc.), ya que los agentes son considerados entidades que pueden interactuar dinámicamente en espacios multidimensionales (1D, 2D o 3D).

- Tradicionalmente, los modelos de ABS han hecho un buen uso de los mecanismos de abstracción basados en clases provistas por los lenguajes de programación orientados a objetos. ABS se puede beneficiar del uso de UML para representar los modelos. UML es un leguaje de modelamiento visual para representar sistemas orientados a objetos y comúnmente es adoptado para apoyar los modelos de agentes en las fases de diseño y comunicación. La programación

5 Método que empieza con los detalles y sube hasta el nivel conceptual más alto. 
orientada a objetos permite el modelamiento de forma natural de los sistemas de agentes con el uso de clases, como plantillas para los agentes, y los métodos de los objetos para representar los comportamientos de los agentes.

- Existen dos métodos para avanzar el reloj en ABS: el método de avance de tiempo de incremento fijo (o actualización asincrónica) y el método de avance del siguiente evento (o actualización sincrónica). Tradicionalmente se ha usado el primer método, ya que es simple de implementar (no requiere una programación ni una lista de eventos) y facilita el modelamiento del comportamiento natural de los agentes, pues no requiere un coordinador central que lleve la cuenta de cuál es el siguiente evento o una gran cantidad de sincronización entre los agentes. Sin embargo, puede ser inexacto y por ello es clave determinar el tamaño adecuado del incremento. El segundo método precisa que el entorno y la duración de las acciones del agente sean determinadas con anticipación. Sin embargo, debido a que pueden ocurrir muchas interacciones, puede ser necesario cancelar y reprogramar ciertos eventos que ya han sido puestos en la lista de eventos que ocurrirán en el futuro. Esto también puede traer ineficiencias en la aplicación del método.

\subsection{Cuadro comparativo de los métodos de simulación}

La tabla 1 contiene una recopilación de los elementos distintivos de cada paradigma, expuestos por diversos autores (Marshall et al., 2015b; Behdani, 2012; Tacko y Robinson, 2009). 


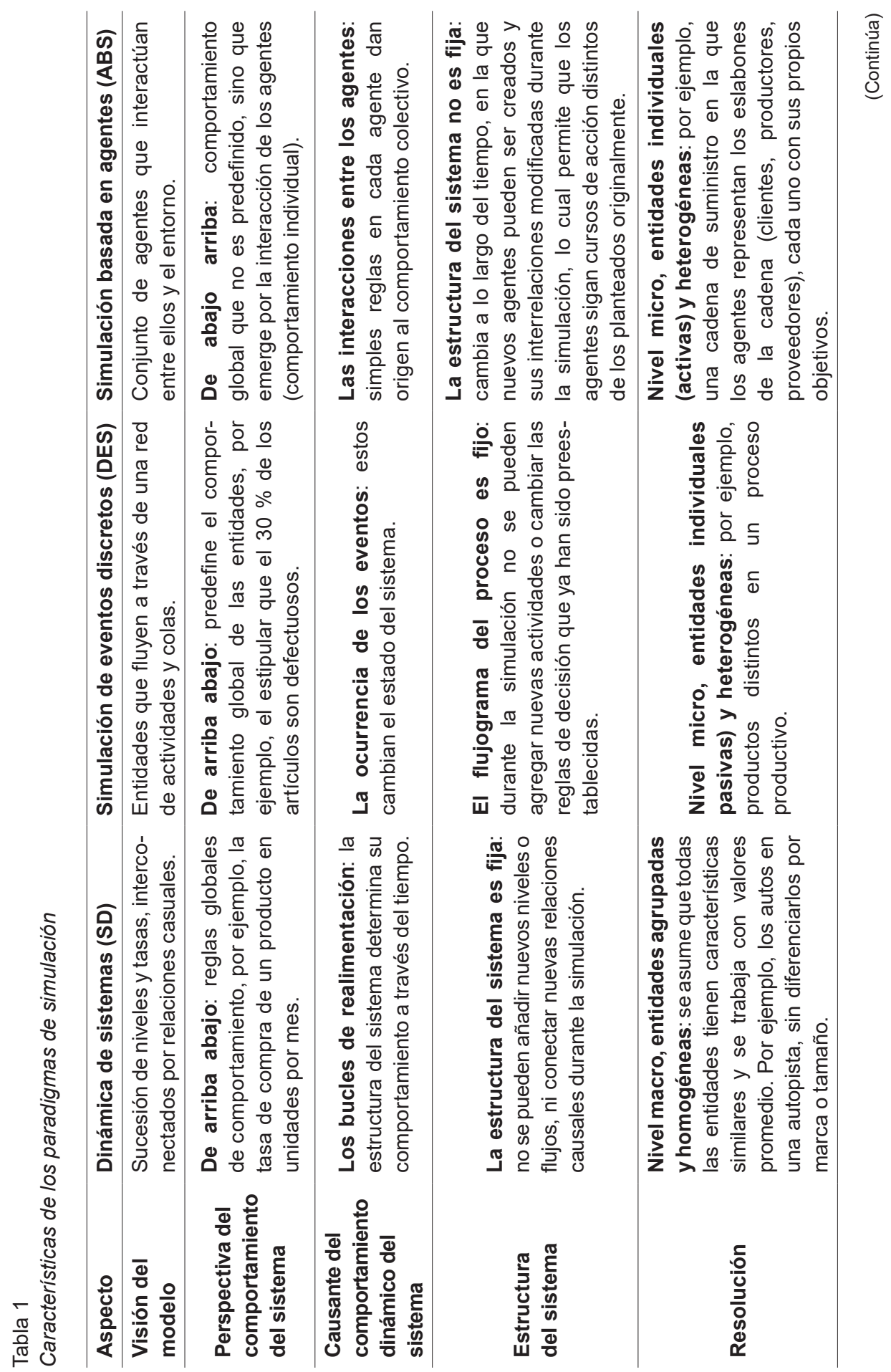




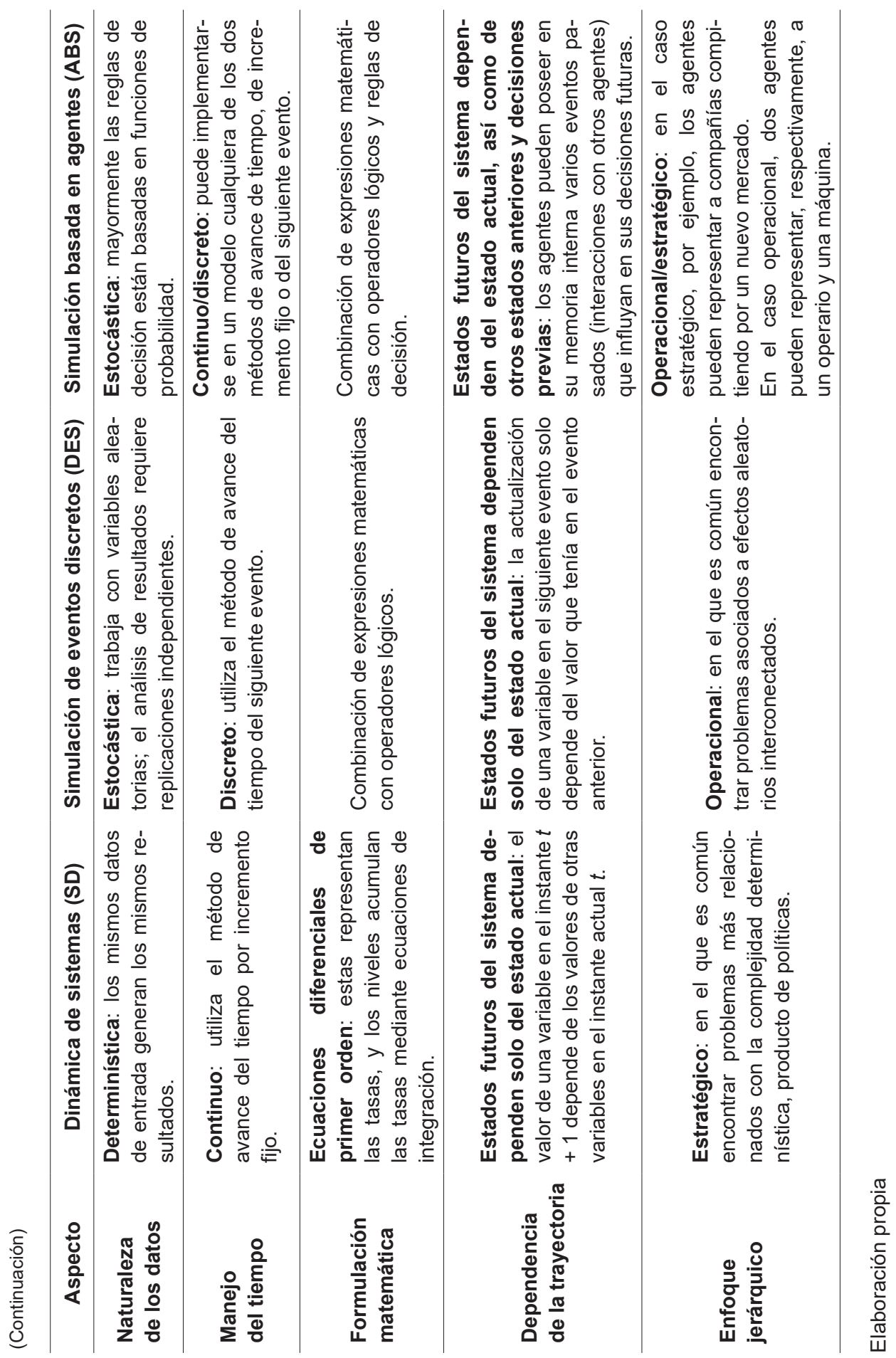




\section{CONCLUSIONES}

En este trabajo se identificaron y compararon las características cualitativas de los tres paradigmas de la simulación: dinámica de sistemas, simulación de eventos discretos y simulación basada en agentes.

La idea esencial en dinámica de sistemas es que todos los elementos en un sistema interactúan a partir de relaciones causales, en las que la estructura del sistema determina su comportamiento dinámico. La estructura del sistema se modela con flujos y bucles de realimentación conectados a variables que definen el estado del sistema. Esta técnica se usa, generalmente, para describir los sistemas del mundo real con una visión macroscópica de arriba abajo, por medio de relaciones no lineales entre entidades consideradas homogéneas y representadas de forma agregada.

La simulación de eventos discretos representa el mundo como un conjunto de entidades que fluyen a través de una red de colas y actividades, en donde los recursos son compartidos entre las actividades. Es muy útil para problemas que consisten en simulaciones de colas o redes complejas con colas, en las que los procesos pueden ser bien definidos y el énfasis se pone en representar incertidumbre a partir de distribuciones estocásticas. Los modelos son típicamente de bucle abierto (openloop) y por ello la retroalimentación no juega un rol preponderante. Las entidades se consideran objetos pasivos y la secuencia de las entidades dentro del modelo está dominada por reglas globales preestablecidas. Se requiere el uso de métodos estadísticos para el análisis de los datos de entrada y los resultados.

La simulación basada en agentes es un método de abajo hacia arriba, es decir, la interacción entre los agentes determina el comportamiento del sistema. El componente básico usado en la construcción de un modelo es el agente, el cual es una entidad activa, ya que puede tomar decisiones autónomas no preestablecidas, es decir, sin un controlador central. Es el método adecuado para cuando no se conocen las reglas globales que rigen el sistema y estas deben emerger del comportamiento individual.

Finalmente, teniendo en cuenta la visión y el enfoque de cada paradigma, además de las características del problema que se quiere representar, se debe elegir el método de simulación adecuado para construir el modelo correspondiente. 


\section{REFERENCIAS}

Angerhofer, B. J., y Angelides, M. C. (2000). System Dynamics Modeling in Supply Chain Management: Research View. En Proceedings of the 2000 Winter Simulation Conference. Orlando: FL.

Behdani, B. (2012). Evaluation of Paradigms for Modeling Supply Chains as Complex Sociotechnical Systems. En Proceedings of the 2012 Winter Simulation Conference. Berlín.

Blanchard, B. S. (1991). Systems Engineering Management. Nueva York: John Wiley \& Sons.

Borshchev, A. (2013). The Big Book of Simulation Modeling: Multimethod Modeling With Anylogic 6.0. Illinois: AnyLogic North America.

Borshchev, B., y Filippov, A. (2004). From System Dynamics and Discrete Event to Practical Agent Based Modeling: Reasons, Techniques, Tools. En Proceedings of the 22nd System Dynamics Conference. Oxford.

Brailsford, S. (2014). Theoretical Comparison of Discrete-Event Simulation and System Dynamics. En S. Brailsford, L. Churilov y B. Dangerfield (Eds.), Discrete-event simulation and system dynamics for management decision making (pp. 105-124). Reino Unido: John Wiley \& Sons.

Brailsford, S. C., Desai, S. M., y Viana, J. (2010). Towards the Holy Grail: Combining System Dynamics and Discrete-Event Simulation in Healthcare. En Proceedings of the 2010 Winter Simulation Conference. Baltimore.

Dangerfield, B. (2014). Systems Thinking and System Dynamics: a Premier. En S. Brailsford, L. Churilov y B. Dangerfield (Eds.), Discrete-event Simulation and System Dynamics for Management Decision Making (pp. 26-65). Reino Unido: John Wiley \& Sons.

Forrester, J. W. (1958). Industrial Dynamics: a Mayor Breakthrough for Decision Makers. Harvard Business Review, 36(4), 37-66.

Harrell, C., Ghosh, B. K., y Bowden, R. O. (2004). Simulation Using Promodel. Nueva York: McGraw-Hill. 
Heath, S. K., Brailsford, S. C., Buss, A., y Macal, S. M. (2011). CrossParadigm Simulation Modelling: Challenges and Successes. En Proceedings of the 2011 Winter Simulation Conference. Phoenix.

Karnon, J., Stahl, J., Brennan, A., Caro, J. J., Mar, J., y Möller, J. (2012). Modeling Using Discrete Event Simulation: a Report of the ISPOR-SMDM Modeling Good Research Practices Task Force-4. Value in Health, (15), 821-827.

Kelton, W. D., Sadowski, R. P., y Sturrock, D. T. (2006). Simulation with ARENA. Nueva York: McGraw-Hill.

Kesaraju, V. S., y Ciarallo, F. W. (2012). Integrated Simulation Combining Process-Driven and Event-Driven Models. Journal of Simulation, 6(1), 9-20.

Law, A. M. (2014). Simulation Modeling And Analysis. Nueva York: Mcgraw-Hill.

Macal, C. M., y North, M. J. (2006). Tutorial on Agent-Based Modeling and Simulation Part 2: How to Model with Agents. En Proceedings of the 2006 Winter Simulation Conference. Monterrey.

Marshall, D. A., Burgos-Liz, L., y Ijzerman, M. J. (2015a). Applying Dynamic Simulation Modeling Methods In Health Care Delivery Research-The SIMULATE Checklist: Report Of The ISPOR Simulation Modeling Emerging Good Practices Task Force. Value in Health, (18), 5-16.

Marshall, D. A., Burgos-Liz, L., Ijzerman, M. J., Crown, W., Padula, W. V., Wong, P. K.,... y Nathaniel, D. O. (2015b). Selecting a Dynamic Simulation Modeling Method for Health Care Delivery Research-Part 2: Report Of The ISPOR Dynamic Simulation Modeling Emerging Good Practices Task Force. Value in Health, (18), 147-160.

Morlán, I. (2010). Modelo de dinámica de sistemas para la implantación de tecnologías de la información en la gestión estratégica universitaria (tesis doctoral). Universidad del País Vasco, España. Recuperada de www.ehu.eus/i.morlan/tesis/memoria/ TesisIMcompleta.pdf

Pegden, D. (2010). Advanced Tutorial: Overview of Simulation World Views. En Proceedings Of The 2010 Winter Simulation Conference. Baltimore. 
Pidd, M. (2004). Computer Simulation in Management Science. Nueva York: John Wiley \& Sons.

Rabelo, L., Helal, M., Jones, A., Min, J., Son, Y. J., y Deshmukh, A. (2003). A Hybrid Approach to Manufacturing Enterprise Simulation. In Proceedings of the 2003 Winter Simulation Conference. Nueva Orleans.

Richardson, G. (1995). Loop Polarity, Loop Dominance, and the Concept of Dominant Polarity. System Dynamics Review, 11(1), 67-88.

Robinson, S. (2004). Simulation: the Practice of Model Development and Use. Reino Unido: John Wiley \& Sons.

Robinson, S. (2014). Discrete-Event Simulation: a Premier. En S. Brailsford, L. Churilov y B. Dangerfield (Eds.), Discrete-event Simulation and System Dynamics for Management Decision Making (pp. 10-25). Reino Unido: John Wiley \& Sons.

Salamon, T. (2011). Design of Agent-Based Models. República Checa: Tomas Bruckner Publishing.

Sarmiento-Vásquez, A.-T. (2016). Análisis comparativo de los paradigmas de simulación (tesis para optar el título de ingeniero industrial). Universidad de Lima.

Schieritz, N., y Milling, P. (2003). Modeling the Forest or Modeling the Trees - A Comparison of System Dynamics and AgentBased Simulation. En Proceedings of the 21st System Dynamics Conference. Nueva York.

Serova, E. (2013). The Role of Agent Based Modelling in the Design of Management Decision Processes. The Electronic Journal Information Systems Evaluation, 16(1), 71-80.

Siebers, P. O., Macal, C. M., Garnet, J., Buxton, D., y Pidd, M. (2010). Discrete-Event Simulation is Dead, Long Live Agent-Based Simulation! Journal of Simulation, 4(3), 204-210.

Skoogh, A., y Johansson, B. (2008). A Methodology for Input Data Management in Discrete Event Simulation Projects. In Proceedings of the 2008 Winter Simulation Conference. Miami.

Sterman, J. (2000). Business Dynamics: Systems Thinking and Modeling for a Complex World. Boston: McGraw-Hill. 
Sumari, S., Ibrahim, R., Zacaria, N. H., y Ab Hamid, A. H. (2013). Comparing Three Simulation Model Using Taxonomy: System Dynamic Simulation, Discrete Event Simulation And Agent Based Simulation. International Journal of Management Excellence, 1(3), 54-59.

Tacko, A. A., y Robinson, S. (2009). Comparing Model Development in Discrete Event Simulation and System Dynamics. En Proceedings of the 2009 Winter Simulation Conference, Austin.

Vangheluwe, H. (2001). Discrete Event Modelling and Simulation. Recuperado de http://www.cs.mcgill.ca/ hv/classes/MS/discrete Event.pdf

Yu, T. T. (2008). The Development of A Hybrid Simulation Modelling Approach Based on Agents and Discrete-Event Modelling (tesis doctoral). Universidad de Southampton, Inglaterra. Recuperada de https://eprints.soton.ac.uk/72111/ 